\title{
Interactive comment on "Optimizing the detection, ablation and ion extraction efficiency of a single particle laser ablation mass spectrometer for application in environments with low aerosol particle concentrations" by Hans-Christian Clemen et al.
}

Giuseppe Petrucci (Referee)

giuseppe.petrucci@uvm.edu

Received and published: 9 July 2020

This is a well written manuscript describing thorough experimental results for a significantly improved method of measuring INPs. Fig. 10: I don't understand why there are two distinct branches for aerosol generation. Please elaborate How robust are the fits to equations 4 and 5 ? There are so many adjustable parameters in each, I wonder whether similarly good fits could be obtained with diverse sets of fitted parameters? In 
other words, is the fit a true global minimum in error or could there possibly be several local minima? Some of the figures don't use symbols (lines are different colors) or use the same symbol (but, again, lines of different colors) to distinguish between experiments. This may be problematic for someone who is visually impaired or doesn't have access to a color version of the manuscript. I would suggest using different symbols for each experiment to facilitate understanding the figures. I found the use of "coincidences" terminology to be confusing. In my experience with this field, "coincidence" is typically reserved for when a second particle enters the timing region before the first particle has passed through second detection laser for estimating particle aerodynamic diameter. In other words, I have always seen this word used to describe a possible experimental complication or error. Sec. 4.2.1 is unclear to me, although I am not familiar with these types of counting instruments. Why sum OPC size channels from 0.65 to $3.0 \mu \mathrm{m}$ to represent a smaller range of particle sizes (1.8 to $2.6 \mu \mathrm{m})$ ? Similarly, for particles greater than $2.6 \mu \mathrm{m}$ ? The authors refer several times to a size-dependent particle beam shift. Why is that? If particles are focused onto the ALS central axis, what causes beam pointing differences for different diameters?

Interactive comment on Atmos. Meas. Tech. Discuss., doi:10.5194/amt-2020-181, 2020. 